\title{
PAPER Marginalized Particle Filter for Blind Signal Detection with Analog Imperfections
}

\author{
Yuki YOSHIDA $^{\dagger \text { a) }}$, Student Member, Kazunori HAYASHI ${ }^{\dagger}$, Member, Hideaki SAKAI $^{\dagger}$, Fellow, \\ and Wladimir BOCQUET ${ }^{\dagger \dagger}$, Member
}

\begin{abstract}
SUMMARY Recently, the marginalized particle filter (MPF) has been applied to blind symbol detection problems over selective fading channels. The MPF can ease the computational burden of the standard particle filter $(\mathrm{PF})$ while offering better estimates compared with the standard PF. In this paper, we investigate the application of the blind MPF detector to more realistic situations where the systems suffer from analog imperfections which are non-linear signal distortion due to the inaccurate analog circuits in wireless devices. By reformulating the system model using the widely linear representation and employing the auxiliary variable resampling (AVR) technique for estimation of the imperfections, the blind MPF detector is successfully modified to cope with the analog imperfections. The effectiveness of the proposed MPF detector is demonstrated via computer simulations.
\end{abstract}

key words: particle filter, blind signal detection, IQ imbalance, CFO

\section{Introduction}

Particle filtering (PF) methods have become a popular class of algorithm to solve non-linear and non-Gaussian estimation problems numerically in an online manner [1]-[3]. While PF is fairly easy to implement, the main drawback is its high computational complexity. One remedy is to analytically marginalize out the state by exploiting the linearity and Gaussianity in the dynamics. The resulting PF is called as marginalized PF (MPF), also known as Kalman PF [3] or Rao-Blackwellised PF [4], and offers better estimates, i.e., estimates with reduced variance, with reduced computational complexity compared with the standard $\mathrm{PF}^{*}$ in some class of problems [5], [6]. Recently, the MPF has been applied to the blind signal detection problem over selective fading channels [7]. By marginalizing out the unknown channel parameters, the MPF detector (MPFD) can obtain the maximum a posteriori (MAP) estimate of the transmitted sequence without explicit channel estimation. In [8], [9], we can see the outstanding performance of the MPFD compared with other blind approaches, such as using a per survivor processing.

Meanwhile, one performance limiting issue in the implementation of wireless communications systems is impairments caused by imperfect analog devices. In most cases,

\footnotetext{
Manuscript received April 20, 2009.

Manuscript revised September 9, 2009.

${ }^{\dagger}$ The authors are with the Graduate School of Informatics, Kyoto University, Kyoto-shi, 606-8501 Japan.

${ }^{\dagger \dagger}$ The author is with France Telecom R\&D, Tokyo Laboratory, Tokyo, 160-0022 Japan.

a)E-mail: yyoshida@sys.i.kyoto-u.ac.jp

DOI: 10.1587/transcom.E93.B.336
}

such impairments cannot be efficiently or entirely eliminated in the analog domain due to the limitations on power consumption, size and cost of devices. Hence, there has been a strong demand for efficient compensation techniques in the digital baseband domain [10]. Significant examples of such imperfections are carrier frequency offset (CFO) and In-phase/Quadrature-phase imbalance (IQI) [11]. They are introduced at the up/down frequency conversions at the transmitter/receiver. The IQI is misalignment between the I- and Q-paths and the CFO is mismatch of frequencies between the local oscillators (LOs) at the transceivers. Practically, degrees of such imperfections differ from devices and are unknown to the receiver.

In this paper, we develop a particle based blind signal detection method in the presence of the transmitter (Tx) and receiver $(\mathrm{Rx}) \mathrm{IQIs}$, and the CFO. Under these imperfections, the state-space representation of the problem results in nonlinear and non-Gaussian and the conventional MPFD cannot be applied. The standard PF approach is applicable to the situation however it is unattractive to lose the computational efficiency of the MPF approach due to analog component imperfections while nothing changes on the physical channel which is the dominant factor of the signal distortion in radio propagation. Therefore some efficient adaptation techniques for the MPFD are worth considering. We first resort to the widely linear (WL) [12] modeling of the problem which enables to deal with improper complex-valued signals [13] due to the IQIs. Based on the WL representation, we show how to adopt the MPF for the case where the IQIs and the CFO parameters are a priori known to the receiver. Next, we propose the imperfection parameter estimation method utilizing the auxiliary variable resampling (AVR) technique [14], [15]. The AVR has been originally proposed to cope with the essential weakness of PF, i.e., performance degradation due to the existence of outliers. Here, we use the technique for a different purpose, i.e., to generate sample estimate of the imperfection parameters by exploiting particles of the previous time step. Finally, by combining the WL modeling and the AVR, the computationally efficient MPF is applied to the detection problem under the analog imperfections. The performance improvement of the proposed MPFD is demonstrated via computer simulations. Moreover, the computational efficiency of the

${ }^{*}$ In this paper, we refer the PF methods without the marginalization as the standard PF to distinct it from the MPF. 
proposed MPFD over the standard PF approach is evaluated experimentally.

This paper is arranged as follows. We provide a brief introduction of PF and the conventional MPFD in Sect. 2. In Sect. 3, we propose an MPFD in the presence of the Tx/Rx IQIs and the CFO. The results of the computer simulations and conclusions appear in Sect. 4 and Sect. 5.

Notations used in the paper are as follows: Vectors or matrices are indicated in bold letters respectively. Superscripts $*, \mathrm{~T}$, and $\mathrm{H}$ represent conjugate, transpose, and Hermitian transpose, respectively. In addition, $|\cdot|$ denotes the determinant of a matrix, $\mathbf{I}_{L}$, an $L \times L$ identity matrix and $\mathbf{0}_{I \times J}$ is an $I \times J$ all zero matrix. We use $x_{i: j}:=\left\{x_{i}, x_{i+1}, \cdots x_{j}\right\}$ for any sequence $x_{i}$, and $p[x \mid y]$ stands for a conditional probability or a conditional probability density/mass function (PDF/PMF) for a continuous/discrete random variable $x$ for given $y$.

\section{Backgrounds}

\section{$2.1 \quad$ Fundamentals of Particle Filtering}

First we briefly introduce PF in the context of sequential importance sampling (SIS) and resampling [16].

Suppose that state $x_{t}$ is a first-order Markov process with given initial PDF and transition PDF $p\left[x_{t} \mid x_{t-1}\right]$ while the observation $y_{t}$ is conditionally independent given $x_{t}$ with the marginal PDF or likelihood $p\left[y_{t} \mid x_{t}\right]$, where $p\left[x_{t} \mid x_{t-1}\right]$ and $p\left[y_{t} \mid x_{t}\right]$ can be computed at least for given samples of $x_{t}$ and $x_{t-1}$, respectively. We consider sequential estimation of the state sequence $x_{0: t}$ based on the current observations $y_{0: t}$. From Bayesian perspective, any estimate on $x_{0: t}$ relies on the posterior PDF $p\left[x_{0: t} \mid y_{0: t}\right]$, e.g., the MAP estimate $x_{0: t}^{\mathrm{MAP}}=\arg \max _{x_{0: t}} p\left[x_{0: t} \mid y_{0: t}\right]$. In order to achieve sequential estimation of the state, one can resort to the following recursion of the posterior:

$$
p\left[x_{0: t} \mid y_{0: t}\right]=\frac{p\left[y_{t} \mid x_{t}\right] p\left[x_{t} \mid x_{t-1}\right]}{p\left[y_{t} \mid y_{t-1}\right]} p\left[x_{0: t-1} \mid y_{0: t-1}\right] .
$$

However, the closed-form expression of the recursion is often intractable [3], e.g., the denominator cannot typically be obtained analytically. PF provides a flexible framework to obtain a Monte-Carlo approximate of the posterior sequentially and recursively based on the SIS algorithm.

The idea behind the SIS is the importance sampling (IS) [17]. Suppose that $M$ samples $\left\{x_{0: t}^{(i)}\right\}_{i=1}^{M}$, which are also called as particles, are drawn from an importance function (IMF) $q\left[x_{0: t} \mid y_{0: t}\right]$ which is designed to have the same support of the posterior PDF while the IMF is much easier to sample from. The IS provides the approximate of the posterior as

$$
p\left[x_{0: t} \mid y_{0: t}\right] \approx \sum_{i=1}^{M} w_{t}^{(i)} \delta_{i}
$$

where $\delta_{i}=1$ if $x_{0: t}=x_{0: t}^{(i)}$ and $\delta_{i}=0$ otherwise, and $w_{t}^{(i)}(>0)$ is the normalized importance weight given by

$$
w_{t}^{(i)} \propto \frac{p\left[x_{0: t} \mid y_{0: t}\right]}{q\left[x_{0: t} \mid y_{0: t}\right]} \text { with } \sum_{i=1}^{M} w_{t}^{(i)}=1 .
$$

In the SIS algorithm, in order to sequentially obtain such weighted particles $\left\{x_{0: t}^{(i)}, w_{t}^{(i)}\right\} \sim p\left[x_{0: t} \mid y_{0: t}\right]$ from existing $\left\{x_{0: t-1}^{(i)}, w_{t-1}^{(i)}\right\} \sim p\left[x_{0: t-1} \mid y_{0: t-1}\right]$ with an arrival of new observation $y_{t}$, we consider the IMF in the form

$$
q\left[x_{0: t} \mid y_{0: t}\right] \propto q\left[x_{t} \mid x_{0: t-1}^{(i)}, y_{0: t}\right] q\left[x_{0: t-1}^{(i)} \mid y_{0: t-1}\right] .
$$

This yields the sequential sampling of each $x_{t}^{(i)}$ without modifying $x_{0: t-1}^{(i)}$. Furthermore, the weights to form (2) can be recursively evaluated by substituting the recursion (1) in (3) as

$$
w_{t}^{(i)} \propto \frac{p\left[y_{t} \mid x_{t}^{(i)}\right] p\left[x_{t}^{(i)} \mid x_{t-1}^{(i)}\right]}{q\left[x_{t}^{(i)} \mid x_{0: t-1}^{(i)}, y_{0: t}\right]} w_{t-1}^{(i)} .
$$

Once the set $\left\{x_{0: t}^{(i)}, w_{t}^{(i)}\right\}$ are generated, any estimate on $x_{0: t}$ at time $t$ can be approximately obtained, e.g., the particle based MAP estimate $\hat{s}_{0: t}^{\mathrm{MAP}}=s_{0: t}^{(\text {imax) }}$ where $\operatorname{imax}=\arg _{i} \max w_{t}^{(i)}$. The procedure of PF at time $t$ is summarized as follows:

With the particles $\left\{x_{0: t-1}^{(i)}, w_{t-1}^{(i)}\right\} \sim p\left[x_{0: t-1} \mid r_{0: t-1}\right]$

[SIS Step]

Sample $x_{t}^{(i)} \sim q\left[x_{t} \mid x_{0: t-1}^{(i)}, y_{0: t}\right]$,

Update weights $\tilde{w}_{t}^{(i)}=\frac{p\left[y_{t} \mid x_{t}^{(i)}\right] p\left[x_{t}^{(i)} \mid x_{t-1}^{(i)}\right]}{q\left[x_{t}^{(i)} \mid x_{0: t-1}^{(i)}, y_{0: t}\right]} w_{t-1}^{(i)}$,

Normalize weights $w_{t}^{(i)}=\frac{\tilde{w}_{t}^{(i)}}{\sum_{i=1}^{M} \tilde{w}_{t}^{(i)}}$,

[Resampling Step]

Sample $j^{(i)}$ from $\{1, \cdots, M\}$ with probability $p[j]=w_{t}^{(i)}$

Replace $\left\{x_{0: t}^{(i)}, w_{t}^{(i)}\right\}$ by $\left\{x_{0: t}^{\left(j^{(i)}\right)}, \frac{1}{M}\right\}$

In the procedure, the resampling step is also included which is commonly employed in PF to mitigate a drawback of the SIS, namely weight degeneracy where all but one of the weights are close to zero [16]. Resampling controls the weights by repropagating the particles according to the multinomial distribution of the weights $\left\{w_{t}^{(i)}\right\}$ so as to construct a new uniformly weighted approximation

$$
p\left[x_{0: t} \mid y_{0: t}\right] \approx \frac{1}{M} \sum_{i=1}^{M} \delta_{i}
$$

The choice of the IMF $q\left[x_{t} \mid x_{0: t-1}^{(i)}, y_{0: t}\right]$ is essential because it determines performance as well as complexity of the SIS algorithm. Two major choices are prior and posterior IMFs:

$$
\text { Prior IMF: } q\left[x_{t} \mid x_{0: t-1}^{(i)}, y_{0: t}\right]=p\left[x_{t} \mid x_{t-1}^{(i)}\right],
$$




$$
\text { Posterior IMF: } \begin{aligned}
& q\left[x_{t} \mid x_{0: t-1}^{(i)}, y_{0: t}\right]=p\left[x_{t} \mid x_{0: t-1}^{(i)}, y_{0: t}\right] \\
& =\frac{p\left[y_{t} \mid x_{t}\right] p\left[x_{t} \mid x_{t-1}^{(i)}\right]}{\int p\left[y_{t} \mid x_{t}\right] p\left[x_{t} \mid x_{t-1}^{(i)}\right] d x_{t}}
\end{aligned}
$$

The prior IMF is much easier to sample from but often results in poor estimates, since no information is exploited from the observation. On the contrary, the use of the posterior IMF is known as optimal in the sense that it minimizes the variance of the importance weights [16], but it is generally impossible to evaluate (8) analytically. In addition, the SIS algorithm based on the prior IMF results in the wellknown bootstrap filter [1].

\subsection{Blind MPFD}

Next, we introduce the conventional MPFD based on the SIS framework.

Suppose the transmitted signals $s_{t}(t=0,1, \cdots, T)$ are i.i.d. uniform random variables, i.e., $s_{t} \sim \mathcal{U}(\mathcal{A})$ where $\mathcal{A}=$ $\left\{a_{n}\right\}_{n=1}^{N}$ denotes a given complex-valued signal constellation. By assuming that the channel coefficients $h(t)(t=0, \cdots L-$ 1) are time-invariant for a duration of the frame $T+1$, the received signal $r_{t}$ is given by

$$
r_{t}=\sum_{l=0}^{L-1} h(l) \hat{s}_{t-l}+v_{t}=\mathbf{h}^{\mathrm{T}} \mathbf{s}_{t}+v_{t}
$$

where $\mathbf{s}_{t}=\left[s_{t} \cdots s_{t-L+1}\right]^{\mathrm{T}}$ (here we set $s_{t}=0(t<0)$ for notational convenience), $\mathbf{h}=[h(0) \cdots h(L-1)]^{\mathrm{T}}$ and $v_{t}$ is the proper complex Gaussian noise, i.e., $v_{t} \sim \mathcal{N}_{c}\left(0, \sigma^{2}\right)$ [13]. We have the state-space representation of the system as

$$
\begin{aligned}
& \text { State: } \mathbf{s}_{t}=\mathbf{T} \mathbf{s}_{t-1}+\mathbf{u}_{t}, \\
& \text { Observation: } r_{t}=\mathbf{h}^{\mathrm{T}} \mathbf{s}_{t}+v_{t},
\end{aligned}
$$

where $\mathbf{T}$ is an $L \times L$ matrix given by

$$
\mathbf{T}:=\left[\begin{array}{c}
\mathbf{0}_{1 \times L} \\
\mathbf{I}_{L-1} \mathbf{0}_{L-1 \times 1}
\end{array}\right],
$$

and $\mathbf{u}_{t}=\left[\begin{array}{llll}s_{t} & 0 & \cdots & 0\end{array}\right]^{\mathrm{T}}$. At time $T$, the main objective is to detect $s_{0: T}$ based on the MAP criterion

$$
s_{0: T}^{\mathrm{MAP}}=\arg \max _{s_{0: T}}\left\{p\left[s_{0: T} \mid r_{0: T}\right]\right\},
$$

with out concerning $\mathbf{h}$.

The SIS approach requires to compute the likelihood $p\left[r_{t} \mid s_{0: t}^{(i)}, r_{0: t-1}\right]$ at least for given $s_{0: t}^{(i)}$, however it is impossible due to the unknown $\mathbf{h}$ in this time. One simple remedy is to jointly propagate sample estimate $\mathbf{h}_{t}^{(i)}$ of $\mathbf{h}$ so as to $\left\{s_{0: t}^{(i)}, \mathbf{h}_{t}^{(i)}, w_{t}^{(i)}\right\} \sim p\left[s_{0: t}, \mathbf{h} \mid r_{0: t}\right]$. Obviously, the posterior IMF is intractable and hence the prior IMFs, say $p\left[s_{t} \mid s_{t-1}\right]$ and $p\left[\mathbf{h}_{t} \mid \mathbf{h}_{t-1}\right]$ might be employed. We refer such approach as the standard PF detector (PFD).

When the observation equation has a linear and Gaussian substructure of the unknowns, that is our case, a more sophisticated approach is to use the posterior IMF by marginalized out the unknowns analytically, namely MPF. Since $p\left[s_{t} \mid s_{t-1}\right]=\mathcal{U}(\mathcal{A})$, the posterior IMF can be computed from the likelihood $p\left[y_{t} \mid x_{t}^{(i)}\right]$ and it can be written as

$$
\begin{aligned}
p\left[r_{t} \mid s_{0: t}, r_{0: t-1}\right] & \\
& =\int p\left[r_{t} \mid \mathbf{h}, s_{0: t}, r_{0: t-1}\right] p\left[\mathbf{h} \mid s_{0: t}, r_{0: t-1}\right] d \mathbf{h} \\
& =\int \mathcal{N}_{c}\left(r_{t} \mid \mathbf{h}^{\mathrm{T}} \mathbf{s}_{t}, \sigma^{2}\right) \times p\left[\mathbf{h} \mid s_{0: t-1}, r_{0: t-1}\right] d \mathbf{h} .
\end{aligned}
$$

Hence, by assuming the Gaussian prior $\mathbf{h} \sim \mathcal{N}_{c}\left(\mathbf{h}_{-\mathbf{1}}, \mathbf{R}_{-1}\right)$, the integrand in (13) is inductively shown to be Gaussian and has a well-known closed-form expression [17]. Such marginalization is also known in the context of the Mixture Kalman filter [18]. The posterior IMF is then obtained from the likelihood as

$$
q\left[s_{t} \mid s_{0: t-1}^{(i)}, r_{0: t}\right]=\frac{p\left[r_{t} \mid s_{t}, s_{0: t-1}^{(i)}, r_{0: t-1}\right]}{\sum_{n=1}^{N} p\left[r_{t} \mid s_{t}=a_{n}, s_{0: t-1}^{(i)}, r_{0: t-1}\right]} .
$$

and the weight update is give by

$$
w_{t}^{(i)} \propto w_{t-1}^{(i)} \sum_{n=1}^{N} p\left[r_{t} \mid s_{t}=a_{n}, s_{0: t-1}^{(i)}, r_{0: t-1}\right] .
$$

The use of the optimum IMF leads to a better estimate than the standard PFD. Moreover, the MPFD can avoid the computational burden to generate additional particles $\mathbf{h}_{t}^{(i)}[6]$.

\section{Proposed Blind MPFD in the Presence of Analog Im- perfections}

\subsection{Problem Formulation}

The input-output relation in (9) implicitly assumes the ideal response of radio devices. Here we reformulate it by taking the Tx/Rx IQIs and the CFO into consideration which inherent in any practical radio devices.

Let $\epsilon_{\mathrm{tx}}$ denotes the amplitude imbalance and $\phi_{\mathrm{tx}}$ is the phase imbalance between the I and Q branches introduced at the transmitter, complex baseband expression for the IQI effect on the ideal symbol $s_{t}$ is given by [11]:

$$
\begin{aligned}
& \hat{s}_{t}=\left(1+\epsilon_{\mathrm{tx}}\right) \mathfrak{R}\left\{s_{t}\right\} \cos \phi_{\mathrm{tx}}-\left(1+\epsilon_{\mathrm{tx}}\right) \mathfrak{J}\left\{s_{t}\right\} \sin \phi_{\mathrm{tx}} \\
& -j \cdot\left(1-\epsilon_{\mathrm{tx}}\right) \mathfrak{R}\left\{s_{t}\right\} \sin \phi_{\mathrm{tx}}+j \cdot\left(1-\epsilon_{\mathrm{tx}}\right) \mathfrak{J}\left\{s_{t}\right\} \cos \phi_{\mathrm{tx}} \\
& \quad=\alpha s_{t}+\beta s_{t}^{*},
\end{aligned}
$$

where $\mathfrak{R}\{\cdot\}$ and $\mathfrak{J}\{\cdot\}$ denote the real and imaginary parts, respectively, and

$$
\begin{aligned}
& \alpha:=\cos \phi_{\mathrm{tx}}+j \cdot \epsilon_{\mathrm{tx}} \sin \phi_{\mathrm{tx}}, \\
& \beta:=\epsilon_{\mathrm{tx}} \cos \phi_{\mathrm{tx}}-j \cdot \sin \phi_{\mathrm{tx}} .
\end{aligned}
$$

The degree of the IQI is commonly evaluated by an image rejection ratio (IRR) which is

$$
\operatorname{IRR}=\mathrm{E}\left[\left|\alpha s_{t}\right|^{2}\right] / \mathrm{E}\left[\left|\beta s_{t}^{*}\right|^{2}\right]=|\alpha|^{2} /|\beta|^{2} .
$$


With practical imbalance values, the IRR is in the order of 20-30 dB [19] and these IRR levels are insufficient in many practical transceiver with high modulation level. The received signal $\hat{r}_{t}$ before the Rx IQI and the CFO is given by

$$
\hat{r}_{t}=\alpha \mathbf{h}^{\mathrm{T}} \mathbf{s}_{t}+\beta \mathbf{h}^{\mathrm{T}} \mathbf{s}_{t}^{*}+v_{t} .
$$

The time domain effect of the CFO $f[\mathrm{~Hz}]$ on $\hat{r}_{t}$ is a phase rotation $\theta=\exp \{j 2 \pi f\}$ proportional with time. As in [20], when the CFO is present together with the Rx IQI, the resulting baseband signal $r_{t}$ can be written by

$$
r_{t}=\gamma \theta^{t} \hat{r}_{t}+\delta \theta^{-t} \hat{r}_{t}^{*},
$$

where $\gamma$ and $\delta$ are the Rx IQI parameters defined by using the amplitude imbalance $\epsilon_{\mathrm{rx}}$ and the phase imbalances $\phi_{\mathrm{rx}}$, i.e.,

$$
\begin{aligned}
& \gamma:=\cos \phi_{\mathrm{rx}}+j \cdot \epsilon_{\mathrm{rx}} \sin \phi_{\mathrm{rx}}, \\
& \delta:=\epsilon_{\mathrm{rx}} \cos \phi_{\mathrm{rx}}-j \cdot \sin \phi_{\mathrm{rx}} .
\end{aligned}
$$

The Rx IQI level is also represented by IRR $=|\delta|^{2} /|\gamma|^{2}$. Consequently, we have the state-space representation of the problem as

$$
\begin{aligned}
& \text { State: } \mathbf{s}_{t}=\mathbf{T s}_{t-1}+\mathbf{u}_{t}, \\
& \text { Observation: } r_{t}=\alpha \gamma \theta^{t} \mathbf{h}^{\mathrm{T}} \mathbf{s}_{t}+\beta^{*} \delta \theta^{-t} \mathbf{h}^{\mathrm{H}} \mathbf{s}_{t} \\
& \quad+\beta \gamma \theta^{t} \mathbf{h}^{\mathrm{T}} \mathbf{s}_{t}^{*}+\alpha^{*} \delta \theta^{-t} \mathbf{h}^{\mathrm{H}} \mathbf{s}_{t}^{*}+\gamma n_{t}+\delta n_{t}^{*},
\end{aligned}
$$

where $n_{t}:=\theta^{t} v_{t}$ for convenience and it is clear that the rotated version of $v_{t}$ is still circular Gaussian, i.e., $n_{t} \sim$ $\mathcal{N}_{c}\left(0, \sigma^{2}\right)$.

Unknowns of the problem are $\mathbf{s}_{t}, \mathbf{h}$ and analog imperfection parameters $\mathcal{B}:=\left\{\epsilon_{\mathrm{tx}}, \phi_{\mathrm{tx}}, \epsilon_{\mathrm{rx}}, \phi_{\mathrm{rx}}, f\right\}$. Clearly, the existence of $\mathcal{B}$ rules out the application of the conventional MPFD. One hasty solution is the use of standard PFD by employing prior IMFs for each of $s_{t}, \mathcal{B}, \mathbf{h}$ and draw samples as $\left\{s_{0: t}^{(i)}, \mathbf{h}_{t}^{(i)}, \mathcal{B}_{t}^{(i)}, w_{t}^{(i)}\right\} \sim p\left[s_{0: t}, \mathbf{h}, \mathcal{B}_{t}^{(i)}, w_{t}^{(i)} \mid r_{0: t}\right]$, but it is unattractive to lose the efficiency of the MPF.

\subsection{Blind MPFD for Known Analog Imperfections}

Firstly, we deal with the case where $\mathcal{B}$ is a priori known to the receiver. In this case, the posterior IMF is propotional to the likelihood and, as in (13), the likelihood can be written as

$$
\begin{aligned}
p & {\left[r_{t} \mid s_{0: t}, r_{0: t-1}, \mathcal{B}\right] } \\
& =\int p\left[r_{t} \mid \mathbf{h}, \mathbf{s}_{t}, \mathcal{B}\right] \cdot p\left[\mathbf{h} \mid s_{0: t-1}, r_{0: t-1}, \mathcal{B}\right] d \mathbf{h} .
\end{aligned}
$$

For given $\mathbf{s}_{t}$ and $\mathcal{B}$, the observation (24) is not linear and Gaussian of $\mathbf{h}$. However, in fact, it is a WL and improper Gaussian of $\mathbf{h}$ [12], [13]. By stacking signals and their complex conjugate in (24), we have

$$
r_{t}=\left(\left(\left[\begin{array}{cc}
\alpha \gamma \theta^{t} & \beta \gamma \theta^{t} \\
\alpha^{*} \delta \theta^{-t} & \beta^{*} \delta \theta^{-t}
\end{array}\right] \otimes \mathbf{I}_{L}\right)\left[\begin{array}{l}
\mathbf{s}_{t} \\
\mathbf{s}_{t}^{*}
\end{array}\right]\right)^{\mathrm{T}}\left[\begin{array}{c}
\mathbf{h} \\
\mathbf{h}^{*}
\end{array}\right]+\left[\begin{array}{ll}
\gamma & \delta
\end{array}\right]\left[\begin{array}{l}
n_{t} \\
n_{t}^{*}
\end{array}\right]
$$

$$
=\left(\left(\boldsymbol{\Lambda}_{t} \otimes \mathbf{I}_{L}\right) \overline{\mathbf{s}}_{t}\right)^{\mathrm{T}} \overline{\mathbf{h}}+z_{t},
$$

where $\otimes$ denotes the Kronecker product, and

$$
\begin{aligned}
\overline{\mathbf{s}}_{t} & :=\left[\begin{array}{l}
\mathbf{s}_{t} \\
\mathbf{s}_{t}^{*}
\end{array}\right], \overline{\mathbf{h}}:=\left[\begin{array}{c}
\mathbf{h} \\
\mathbf{h}^{*}
\end{array}\right], z_{t}:=\left[\begin{array}{ll}
\gamma & \delta
\end{array}\right]\left[\begin{array}{l}
n_{t} \\
n_{t}^{*}
\end{array}\right], \\
\boldsymbol{\Lambda}_{t} & :=\left[\begin{array}{cc}
\alpha \gamma \theta^{t} & \beta \gamma \theta^{t} \\
\alpha^{*} \delta \theta^{-t} & \beta^{*} \delta \theta^{-t}
\end{array}\right] .
\end{aligned}
$$

Now it is clear that, (26) is a linear system of the extended channel vector $\overline{\mathbf{h}}$ for given $\mathbf{s}_{t}$ and $\mathcal{B}$. As for the noise, $z_{t}$ is no more proper complex Gaussian but improper Gaussian [13], [21]. According to [13], by defining $\overline{\mathbf{z}}_{t}=\left[z_{t} z_{t}^{*}\right]^{\mathrm{T}}$, the $\mathrm{PDF}$ of $z_{t}$ is given by

$$
p\left[z_{t}\right]=p\left[\overline{\mathbf{z}}_{t}\right]=\overline{\mathcal{N}}_{c}\left(\left[\begin{array}{ll}
0 & 0
\end{array}\right]^{\mathrm{T}}, \boldsymbol{\Phi}\right),
$$

where $\overline{\mathcal{N}}_{c}(\cdot)$ denotes the improper complex Gaussian $\mathrm{PDF}^{\dagger}$ and

$$
\boldsymbol{\Phi}=\left[\begin{array}{cc}
\left(\gamma \gamma^{*}+\delta \delta^{*}\right) \sigma^{2} & 2 \gamma \delta \sigma^{2} \\
2 \gamma^{*} \delta^{*} \sigma^{2} & \left(\gamma \gamma^{*}+\delta \delta^{*}\right) \sigma^{2}
\end{array}\right] .
$$

Correspondingly, $p\left[r_{t} \mid \mathbf{h}, \mathbf{s}_{t}, \mathcal{B}\right]$ also becomes an improper complex Gaussian and, by defining $\overline{\mathbf{r}}_{t}=\left[r_{t} r_{t}^{*}\right]^{\mathrm{T}}$, it follows that

$$
p\left[r_{t} \mid \mathbf{h}, \mathbf{s}_{t}, \mathcal{B}\right]=p\left[\overline{\mathbf{r}}_{t} \mid \mathbf{h}, \mathbf{s}_{t}, \mathcal{B}\right]=\overline{\mathcal{N}}_{c}\left(\boldsymbol{\Gamma}_{t} \overline{\mathbf{h}}, \boldsymbol{\Phi}\right),
$$

where

$$
\boldsymbol{\Gamma}_{t}=\left[\begin{array}{c}
\left(\left(\boldsymbol{\Lambda}_{t} \otimes \mathbf{I}_{L}\right) \overline{\mathbf{s}}_{t}\right)^{\mathrm{T}} \\
\left(\left(\boldsymbol{\Lambda}_{t} \otimes \mathbf{I}_{L}\right) \overline{\mathbf{s}}_{t}\right)^{\mathrm{H}} \mathbf{D}
\end{array}\right], \mathbf{D}:=\left[\begin{array}{cc}
\mathbf{0}_{L \times L} & \mathbf{I}_{L} \\
\mathbf{I}_{L} & \mathbf{0}_{L \times L}
\end{array}\right] .
$$

Meanwhile, the posterior of the channel $p\left[\mathbf{h} \mid s_{0: t}, r_{0: t}, \mathcal{B}\right]$ is also proportional to the integrand in (25):

$$
p\left[\mathbf{h} \mid s_{0: t}, r_{0: t}, \mathcal{B}\right] \propto p\left[r_{t} \mid \mathbf{h}, \mathbf{s}_{t}, \mathcal{B}\right] p\left[\mathbf{h} \mid s_{0: t-1}, r_{0: t-1}, \mathcal{B}\right] .
$$

Thus, by assuming $\overline{\mathbf{h}} \sim \overline{\mathcal{N}}_{c}\left(\overline{\mathbf{h}}_{-1}, \overline{\mathbf{R}}_{-1}\right)$ and denoting $\overline{\mathbf{h}}_{t}$ and $\overline{\mathbf{R}}_{t}$ as the mean and covariance of $p\left[\mathbf{h} \mid s_{0: t}, r_{0: t}, \mathcal{B}\right]$, the integrand of (25) can be written as

$$
\begin{aligned}
p[ & \left.r_{t} \mid \mathbf{h}, \mathbf{s}_{t}, \mathcal{B}\right] \cdot p\left[\mathbf{h} \mid s_{0: t-1}, r_{0: t-1}, \mathcal{B}\right] \\
= & \pi^{-1-L}\left|\boldsymbol{\Phi} \overline{\mathbf{R}}_{t-1}\right|^{-\frac{1}{2}} \exp \left\{-\frac{1}{2}\left[\left(\overline{\mathbf{h}}-\overline{\mathbf{h}}_{t}\right)^{\mathrm{H}} \overline{\mathbf{R}}_{t}^{-1}\left(\overline{\mathbf{h}}-\overline{\mathbf{h}}_{t}\right)\right.\right. \\
& \left.\left.+\overline{\mathbf{r}}_{t}^{\mathrm{H}} \boldsymbol{\Phi}^{-1} \overline{\mathbf{r}}_{t}+\overline{\mathbf{h}}_{t-1}^{\mathrm{H}} \overline{\mathbf{R}}_{t-1}^{-1} \overline{\mathbf{h}}_{t-1}-\overline{\mathbf{h}}_{t}^{\mathrm{H}} \overline{\mathbf{R}}_{t}^{-1} \overline{\mathbf{h}}_{t}\right]\right\},
\end{aligned}
$$

where

$$
\begin{aligned}
& \overline{\mathbf{R}}_{t}^{-1}=\boldsymbol{\Gamma}_{t}^{\mathrm{H}} \boldsymbol{\Phi}^{-1} \boldsymbol{\Gamma}_{t}+\overline{\mathbf{R}}_{t-1}^{-1}, \\
& \overline{\mathbf{h}}_{t}=\overline{\mathbf{R}}_{t}\left(\boldsymbol{\Gamma}_{t}^{\mathrm{H}} \boldsymbol{\Phi}^{-1} \overline{\mathbf{r}}_{t}+\mathbf{R}_{t-1}^{-1} \overline{\mathbf{h}}_{t-1}\right) .
\end{aligned}
$$

Consequently, the likelihood can be found as

$$
\begin{aligned}
& \frac{p\left[r_{t} \mid s_{0: t}, r_{0: t-1}, \mathcal{B}\right]=\pi^{-1}\left|\overline{\mathbf{R}}_{t}\right|^{\frac{1}{2}}\left|\mathbf{\Phi} \overline{\mathbf{R}}_{t-1}\right|^{-\frac{1}{2}}}{{ }^{\dagger} \overline{\mathcal{N}}_{c}(\overline{\mathbf{x}} \mid \overline{\boldsymbol{\mu}}, \overline{\mathbf{\Sigma}}):=\pi^{-n}|\overline{\boldsymbol{\Sigma}}|^{-\frac{1}{2}} \exp \left[-\frac{1}{2}(\overline{\mathbf{x}}-\overline{\boldsymbol{\mu}})^{\mathrm{H}} \overline{\boldsymbol{\Sigma}}^{-1}(\overline{\mathbf{x}}-\overline{\boldsymbol{\mu}})\right] \text { where } \mu} \\
& \text { is the mean and } \boldsymbol{\Sigma} \text { is the covariance of } \overline{\mathbf{x}}=\left[\mathbf{x}^{\mathrm{T}} \mathbf{x}^{\mathrm{H}}\right]^{\mathrm{T}} .
\end{aligned}
$$




$$
\times \exp \left\{-\frac{1}{2}\left[\overline{\mathbf{r}}_{t}^{\mathrm{H}} \boldsymbol{\Phi}^{-1} \overline{\mathbf{r}}_{t}+\overline{\mathbf{h}}_{t-1}^{\mathrm{H}} \overline{\mathbf{R}}_{t-1}^{-1} \overline{\mathbf{h}}_{t-1}-\overline{\mathbf{h}}_{t}^{\mathrm{H}} \overline{\mathbf{R}}_{t}^{-1} \overline{\mathbf{h}}_{t}\right]\right\}
$$

Therefore, the MPFD with the posterior IMF

$$
q\left[s_{t} \mid s_{0: t-1}^{(i)}, r_{0: t}, \mathcal{B}\right]=\frac{p\left[r_{t} \mid s_{t}, s_{0: t-1}^{(i)}, r_{0: t-1}, \mathcal{B}\right]}{\sum_{n=1}^{N} p\left[r_{t} \mid s_{t}=a_{n}, s_{0: t-1}^{(i)}, r_{0: t-1}, \mathcal{B}\right]},
$$

and the weight update

$$
w_{t}^{(i)} \propto w_{t-1}^{(i)} \sum_{n=1}^{N} p\left[r_{t} \mid s_{t}=a_{n}, s_{0: t-1}^{(i)}, r_{0: t-1}, \mathcal{B}\right],
$$

is applicable for the systems with known imperfections. We summarize the algorithm in Algorithm 1.

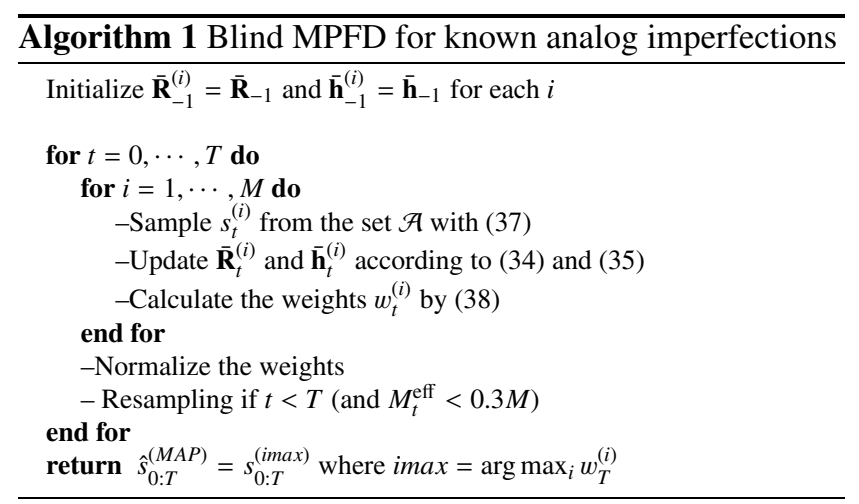

\subsection{Imperfection Parameter Estimation via the AVR}

In order to deal with the unknown imperfection parameters, we consider generating sample estimate $\mathcal{B}_{t}^{(i)}=$ $\left\{\epsilon_{\mathrm{tx} t}^{(i)}, \phi_{\mathrm{tx} t}^{(i)}, \epsilon_{\mathrm{rx} t}^{(i)}, \phi_{\mathrm{rx} t}^{(i)}, f_{t}^{(i)}\right\}$ together with $\left\{s_{0: t}^{(i)}\right\}$. Moreover, to enjoy the computational efficiency of the MPFD proposed in the previous section, we resort to a two stage sampling based on the following decomposition,

$$
p\left[s_{0: t}, \mathcal{B} \mid r_{0: t}\right]=p\left[\mathcal{B} \mid r_{0: t}\right] p\left[s_{0: t} \mid r_{0: t}, \mathcal{B}\right] .
$$

The resulting SIS algorithm to obtain $\left\{s_{0: t}^{(i)}, \mathcal{B}_{t}^{(i)}\right\} \sim$ $p\left[s_{0: t}, \mathcal{B}_{t} \mid r_{0: t}\right]^{\dagger}$ can be written as

$$
\begin{aligned}
\mathcal{B}_{t}^{(i)} & \sim q_{1}\left[\mathcal{B}_{t} \mid r_{0: t}\right], \\
s_{t}^{(i)} & \sim q_{2}\left[s_{t} \mid s_{0: t-1}^{(i)}, \mathcal{B}_{t-1}^{(i)}, r_{0: t}\right], \\
w_{t}^{(i)} & \propto \frac{p\left[r_{t} \mid s_{0: t}^{(i)}, \mathcal{B}_{t}^{(i)}, r_{0: t-1}\right] p\left[\mathcal{B}_{t}^{(i)} \mid \mathcal{B}_{t-1}^{(i)}\right]}{q_{1}\left[\mathcal{B}_{t}^{(i)} \mid r_{0: t}\right] q_{2}\left[s_{t}^{(i)} \mid s_{0: t-1}^{(i)}, \mathcal{B}_{t-1}^{(i)}, r_{0: t}\right]} w_{t-1}^{(i)},
\end{aligned}
$$

where we use the facts $p\left[s_{t}, \mathcal{B}_{t} \mid s_{0: t-1}^{(i)}, \mathcal{B}_{t-1}^{(i)}\right]=p\left[s_{t} \mid s_{0: t-1}^{(i)}\right]$ $p\left[\mathcal{B}_{t} \mid \mathcal{B}_{t-1}^{(i)}\right]$ and $p\left[s_{t} \mid s_{0: t-1}^{(i)}\right]=\mathcal{U}(\mathcal{A})$, and $q_{1}[\cdot]$ and $q_{2}[\cdot]$ represent the IMFs to draw $\mathcal{B}_{t}^{(i)}$ and $s_{t}^{(i)}$, respectively. Clearly, the posterior IMF as in (36) can be used for $q_{2}[\cdot]$.
Now, our interest is the choice of $q_{1}\left[\mathcal{B}_{t} \mid r_{0: t}\right]$. In fact, the posterior $p\left[\mathcal{B}_{t} \mid r_{0: t}\right]$ is intractable. Therefore, we first resort to the discrete approximation of the posterior by exploiting particles and weights at the previous time, i.e.,

$$
\begin{aligned}
& p\left[\mathcal{B}_{t} \mid r_{0: t}\right]= \\
& \int p\left[\mathcal{B}_{t} \mid s_{0: t-1}, \mathcal{B}_{t-1}, r_{0: t}\right] p\left[s_{0: t-1}, \mathcal{B}_{t-1} \mid r_{0: t}\right] d s_{0: t-1} d \mathcal{B}_{t-1} \\
& \approx \frac{1}{M} \sum_{i=1}^{M} p\left[\mathcal{B}_{t} \mid s_{0: t-1}^{(i)}, \mathcal{B}_{t-1}^{(i)}, r_{0: t}\right] p\left[s_{0: t-1}^{(i)}, \mathcal{B}_{t-1}^{(i)} \mid r_{0: t}\right] \\
& \propto \sum_{i=1}^{M} p\left[r_{t} \mid s_{0: t-1}^{(i)}, \mathcal{B}_{t}, r_{0: t-1}\right] p\left[\mathcal{B}_{t} \mid \mathcal{B}_{t-1}^{(i)}\right] w_{t-1}^{(i)} .
\end{aligned}
$$

We then apply the idea of the AVR to sample $\mathcal{B}_{t}^{(i)}$ from this mixture distribution. In the AVR, instead of sampling from the mixture $p\left[\mathcal{B}_{t} \mid r_{0: t}\right]$ directly, we try to perform the sampling in a higher dimension $p\left[\mathcal{B}_{t}, k \mid r_{0: t}\right]$ where the integer $k(k=1, \cdots, M)$ represents to choose the $k$ th factor or stratum of the mixture (43). The integer $k$ is socalled auxiliary variable as it is present simply to aid the task of the simulation and will be discarded later. The IMF $q_{1}\left[\mathcal{B}_{t} \mid r_{0: t}\right]=q\left[\mathcal{B}_{t}, k \mid r_{0: t}\right]$ is designed by mimicking the $k$ th factor of (43) as

$$
q\left[\mathcal{B}_{t}, k \mid r_{0: t}\right] \propto p\left[r_{t} \mid s_{0: t-1}^{(k)}, \hat{\mathcal{B}}_{t}^{(k)}, r_{0: t-1}\right] p\left[\mathcal{B}_{t} \mid \mathcal{B}_{t-1}^{(k)}\right] w_{t-1}^{(k)},
$$

where $\hat{\mathcal{B}}_{t}^{(i)}=\left\{\hat{\epsilon}_{\mathrm{tx} t}^{(i)}, \hat{\phi}_{\mathrm{tx} t}^{(i)}, \hat{\epsilon}_{\mathrm{rx} t}^{(i)}, \hat{\phi}_{\mathrm{rx} t}^{(i)}, \hat{f}_{t}^{(i)}\right\}$ are some characterization of $\mathcal{B}_{t}$ for given $\mathcal{B}_{t-1}^{(i)}$, i.e., the averages, modes or medians of $p\left[\mathcal{B}_{t} \mid \mathcal{B}_{t-1}^{(i)}\right]$. From the factorization

$$
q\left[\mathcal{B}_{t}, k \mid r_{0: t}\right]=q\left[\mathcal{B}_{t} \mid k, r_{0: t}\right] q\left[k \mid r_{0: t}\right],
$$

by defining,

$$
q\left[\mathcal{B}_{t} \mid k, r_{0: t}\right]:=p\left[\mathcal{B}_{t} \mid \mathcal{B}_{t-1}^{(k)}\right],
$$

it follows that

$$
q\left[k \mid r_{0: t}\right] \propto p\left[r_{t} \mid s_{0: t-1}^{(k)}, \hat{\mathcal{B}}_{t}^{(k)}, r_{0: t-1}\right] w_{t-1}^{(k)} .
$$

This means that the samples $\left\{\mathcal{B}_{t}^{(i)}\right\}$ can be obtained by firstly simulating $k$ with probability proportional to $p\left[r_{t} \mid \hat{\mathcal{B}}_{t}^{(k)}\right] w_{t-1}^{(k)}$, and then sampling $\mathcal{B}_{t}^{(i)}$ from $p\left[\mathcal{B}_{t} \mid \mathcal{B}_{t-1}^{(k)}\right]$ for given $k$.

Consequently, from (37) and (44), the overall IMF is given by

$$
\begin{aligned}
& q_{1}\left[\mathcal{B}_{t}, k \mid r_{0: t}\right] q_{2}\left[s_{t} \mid s_{0: t-1}^{\left(k^{(i)}\right)}, \mathcal{B}_{t}^{(i)}, r_{0: t}\right] \\
& \propto p\left[r_{t} \mid s_{0: t-1}^{\left(k^{(i)}\right)}, \hat{\mathcal{B}}_{t}^{\left(k^{(i)}\right)}, r_{0: t-1}\right] p\left[\mathcal{B} \mid \mathcal{B}_{t-1}^{\left(k^{(i)}\right)}\right] w_{t-1}^{\left(k^{(i)}\right)} \\
& \cdot \frac{p\left[r_{t} \mid s_{t}, s_{0: t-1}^{(i)}, r_{0: t-1}, \mathcal{B}_{t}^{(i)}\right]}{\sum_{n=1}^{N} p\left[r_{t} \mid s_{t}=a_{n}, s_{0: t-1}^{(i)}, r_{0: t-1}, \mathcal{B}_{t}^{(i)}\right]}
\end{aligned}
$$

${ }^{\dagger}$ Hereafter $\mathcal{B}$ is denoted by $\mathcal{B}_{t}$ to clarify the correspondence between $\mathcal{B}_{t}^{(i)}$, but surely it is still a set of static unknown model parameters, i.e., $\mathcal{B}_{t}=\mathcal{B}_{t-1}$. 


$$
\begin{gathered}
=\left(\sum_{n=1}^{N} p\left[r_{t} \mid s_{t}=a_{n}, s_{0: t-1}^{\left(k^{(i)}\right)}, \hat{\mathcal{B}}_{t}^{\left(k^{(i)}\right)}, r_{0: t-1}\right]\right) \\
\cdot \frac{p\left[r_{t} \mid s_{t}, s_{0: t-1}^{(i)}, r_{0: t-1}, \mathcal{B}_{t}^{(i)}\right] p\left[\mathcal{B} \mid \mathcal{B}_{t-1}^{\left(k^{(i)}\right)}\right]}{\sum_{n=1}^{N} p\left[r_{t} \mid s_{t}=a_{n}, s_{0: t-1}^{(i)}, r_{0: t-1}, \mathcal{B}_{t}^{(i)}\right]} w_{t-1}^{\left(k^{(i)}\right)}
\end{gathered}
$$

where $k^{(i)}$ denotes the auxiliary variable at the $i$ th particle. Correspondingly, the weights update (42) is obtained as

$$
\begin{gathered}
w_{t}^{(i)} \propto \frac{p\left[r_{t} \mid s_{0: t}^{(i)}, \mathcal{B}_{t}^{(i)}, r_{0: t-1}\right] p\left[\mathcal{B}_{t}^{(i)} \mid \mathcal{B}_{t-1}^{\left(k^{(i)}\right)}\right]}{q_{1}\left[\mathcal{B}_{t}^{(i)} \mid r_{0: t}\right] q_{2}\left[s_{t}^{(i)} \mid s_{0: t-1}^{\left(k^{(i)}\right)}, \mathcal{B}_{t-1}^{(i)}, r_{0: t}\right]} w_{t-1}^{\left(k^{(i)}\right)} \\
=\frac{\sum_{n=1}^{N} p\left[r_{t} \mid s_{t}=a_{n}, s_{0: t-1}^{\left(k^{(i)}\right)}, \mathcal{B}_{t}^{(i)}, r_{0: t-1}\right]}{\sum_{n=1}^{N} p\left[r_{t} \mid s_{t}=a_{n}, s_{0: t-1}^{\left(k^{(i)}\right)}, \hat{\mathcal{B}}_{t}^{\left(k^{(i)}\right)}, r_{0: t-1}\right]}
\end{gathered}
$$

The rest of the problem is the choice of $\hat{\mathcal{B}}_{t}^{\left(k^{(i)}\right)}$ and the transition $p\left[\mathcal{B}_{t} \mid \mathcal{B}_{t-1}^{\left(k^{(i)}\right)}\right]$. Since all the parameters in $\mathcal{B}_{t}$ are static, it is natural to set $\mathcal{B}_{t}^{(i)}=\mathcal{B}_{t-1}^{\left(k^{(i)}\right)}$ and $\hat{\mathcal{B}}_{t}^{\left(k^{(i)}\right)}=\mathcal{B}_{t}^{\left(k^{(i)}\right)}$ without sampling. However, the inability to rejuvenate $\mathcal{B}_{t}^{(i)}$ makes the accuracy of the final estimate greatly sensitive to the initial samples. Thus we introduce some perturbation for each parameter in $\mathcal{B}_{t}^{(i)}$ by employing a kernel smoothing method [2]. For example, for the CFO parameter $f_{t}$ in $\mathcal{B}_{t}$,

$$
\begin{aligned}
& p\left[f_{t} \mid f_{t-1}^{\left(k^{(i)}\right)}\right]=\mathcal{N}\left(\hat{f}_{t}^{\left(k^{(i)}\right)}, \xi^{2} \rho_{t-1}\right), \\
& \hat{f}_{t}^{(i)}=\eta f_{t-1}^{(i)}+(1-\eta) \bar{f}_{t-1},
\end{aligned}
$$

where $\bar{f}_{t-1}$ is a weighted average, i.e., $\bar{f}_{t-1}=\sum_{i=1}^{M} w_{t-1}^{(i)} f_{t-1}^{(i)}$, $\rho_{t-1}$ is a weighted sample covariance and $\mathcal{N}(\cdot)$ denotes real Gaussian PDF. It is suggested in [2] that $\eta=\sqrt{1-\xi^{2}}, \xi^{2}=$ $1-((3 v-1) / 2 v)^{2}$ where $v$ is a discount factor in $(0,1]$, typically around 0.95 to 0.99 . What is more, since the IQI and the CFO will be limited in a certain range by the manufacturer, it would be more practical to use truncated real Gaussian $\mathcal{T} \mathcal{N}\left(\hat{f}_{t}^{\left(k^{(i)}\right)}, \xi^{2} \rho_{t-1}, f_{l}, f_{u}\right)$ in which samples constrained in the region $\left[f_{l}, f_{u}\right]$, instead of $(50)$.

We summarize the algorithm of the proposed MPFD with the AVR in Algorithm 2. It should be mentioned that the algorithm consists only of the SIS step utilizing the IMF (48). In [15], the authors have pointed out that the SIS including AVR possibly avoid the degeneracy without any resampling procedure. Hence, from a view point of computational complexity, we omit a resampling step in the algorithm. In addition, in [22], [23]), the authors have proposed a similar algorithm through the different approach where a suboptimal IMF called hybrid IMF is employed and, to overcome a drawback of the IMF, the AVR is included in the resulting $\mathrm{PF}$.

\section{Simulation Results}

Here, we evaluate the performance of the proposed MPFDs via computer simulations. In our experiments, we have assumed a time-invariant frequency-selective fading channel of length $L=3$ with the prior $p[\mathbf{h}]=\mathcal{N}_{c}\left(\mathbf{h}_{-1}, \mathbf{R}_{-1}\right)$ where

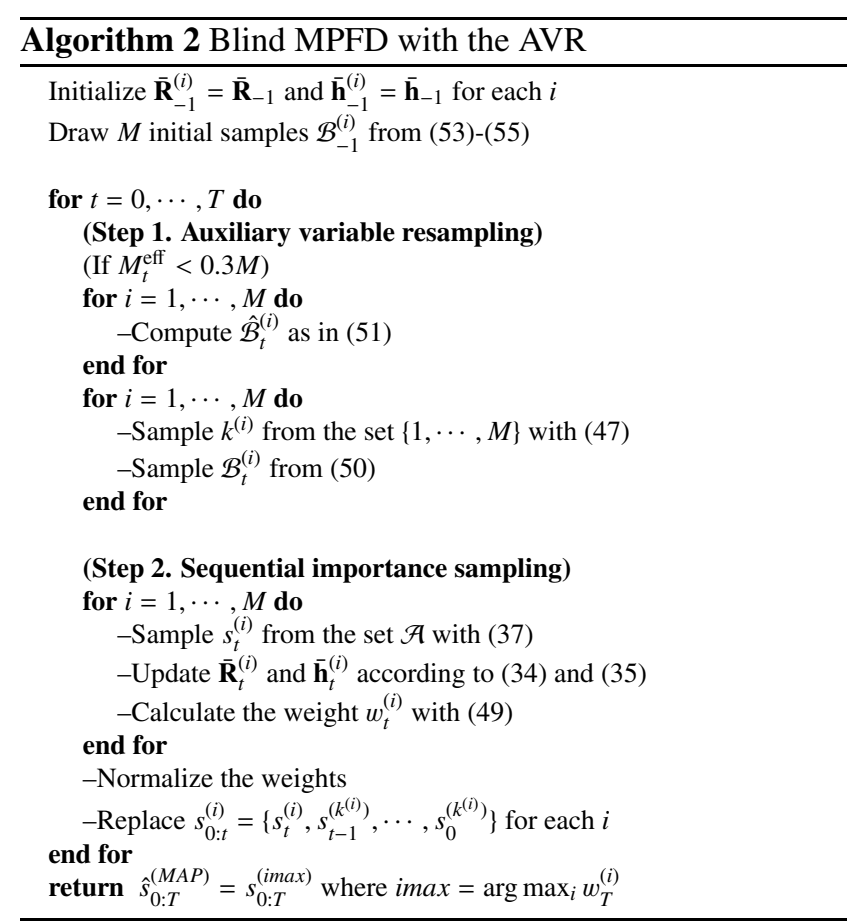

$$
\mathbf{h}_{-1}=\left[\begin{array}{l}
1 \\
0 \\
0
\end{array}\right], \quad \mathbf{R}_{-1}=\left[\begin{array}{ccc}
0.2 & 0 & 0 \\
0 & 0.1 & 0 \\
0 & 0 & 0.05
\end{array}\right]
$$

As for transmitted signal, differentially coded QPSK is employed for modulation scheme to mitigate the phase ambiguity inherent in any blind receiver and we send $T=200$ symbols. Analog imperfection parameters are randomly generated as

$$
\begin{aligned}
& \epsilon_{\mathrm{tx}}, \epsilon_{\mathrm{rx}} \sim \mathcal{T} \mathcal{N}(0,0.01,0,0.1), \\
& \phi_{\mathrm{tx}}, \phi_{\mathrm{rx}} \sim \mathcal{T} \mathcal{N}(0,0.01,-0.05,0.05), \\
& f \sim \mathcal{T} \mathcal{N}(0,0.01,-0.01,0.01),
\end{aligned}
$$

where the resulting $\mathrm{Tx}$ or $\mathrm{Rx}$ IRR are about $30 \mathrm{~dB}$. We mainly test four detectors:

- Conventional MPFD as in Sect. 2.2

- Standard PFD

- Proposed MPFD for known analog imperfections in Algorithm 1

- Proposed MPFD with the AVR in Algorithm 2

As for the standard PFD, the prior IMF is used for $s_{t}$ while, since both $\mathbf{h}_{t}$ and $\mathcal{B}_{t}$ are static, the smoothing kernels are employed to generate $\mathbf{h}_{t}^{(i)}$ and $\mathcal{B}_{t}^{(i)}$ where $v=0.97$. We employ the multinomial resampling technique [1], [24] in three detectors other than the MPFD with the AVR. The number of particles $M=300$ and all the priors are assumed to be known to the receiver. Following results are averages taken over 2000 realizations of channels and analog imperfection parameters.

We first evaluate the performance degradation of the conventional MPFD due to the analog imperfections, and the effectiveness of the proposed MPFDs. Fig. 1 shows the 


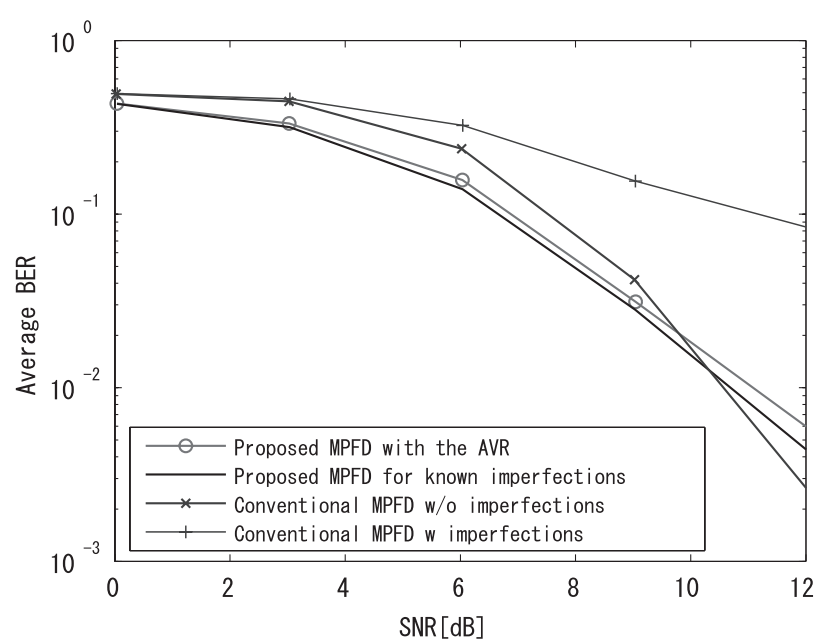

Fig. 1 BER performance versus SNR of the conventional and proposed MPFDs.

bit error rate (BER) performance of the conventional MPFD and the proposed MPFDs versus the transmitted signal-tonoise power ratio (SNR), i.e., SNR $=\mathrm{E}\left[\left|s_{t}\right|^{2} /\left|n_{t}\right|^{2}\right]$. In the figure, the BER performance of the conventional MPFD without analog imperfections is included for comparison purpose. Clearly, the imperfections seriously deteriorate the performance of the conventional MPFD. On the other hand, the MPFD with the AVR can avoid such deterioration and moreover can achieve the comparable performance to the MPFD for known analog imperfections. Fig. 2 shows the root mean square-error (RMSE) of the estimate of the CFO parameter $f$ defined as

$$
\mathrm{RMSE}=\sqrt{\mathrm{E}\left[\left|f-f_{t}^{(i m a x)}\right|^{2}\right]},
$$

versus time $t$ in the MPFD with the AVR where the SNR is $12 \mathrm{~dB}$. The figure shows that the proposed MPFD successfully estimate the CFO parameter through its AVR step. It should be mentioned that, it is difficult to evaluate accuracy of the estimates of the other parameters, because $\left\{\epsilon_{\mathrm{tx}}, \epsilon_{\mathrm{rx}}, \phi_{\mathrm{tx}}, \phi_{\mathrm{rx}}\right\}$ or equivalently $\{\alpha, \beta, \gamma, \delta\}$ have scale ambiguity between $\mathbf{h}$.

In practical PF approaches, one can reduce the computational burden of PF without losing its performance by skipping the resampling step when the degeneracy is not so serious [3], [25]. However, this might not be the case with the proposed MPFD with the AVR since the IMF as in (48) including the AVR step itself. Therefore, we evaluate the BER performance of the MPFD with the AVR with the computational complexity reduction. A well-known measure of degeneracy is the effective sample size [25] which is estimated by

$$
M_{t}^{\mathrm{eff}}=\frac{1}{\sum_{i=1}^{M}\left(w_{t}^{(i)}\right)^{2}} .
$$

Figure 3 shows the BER performances of the conventional MPFD without analog imperfections, the MPFD for known analog imperfection, and the MPFD with the AVR where

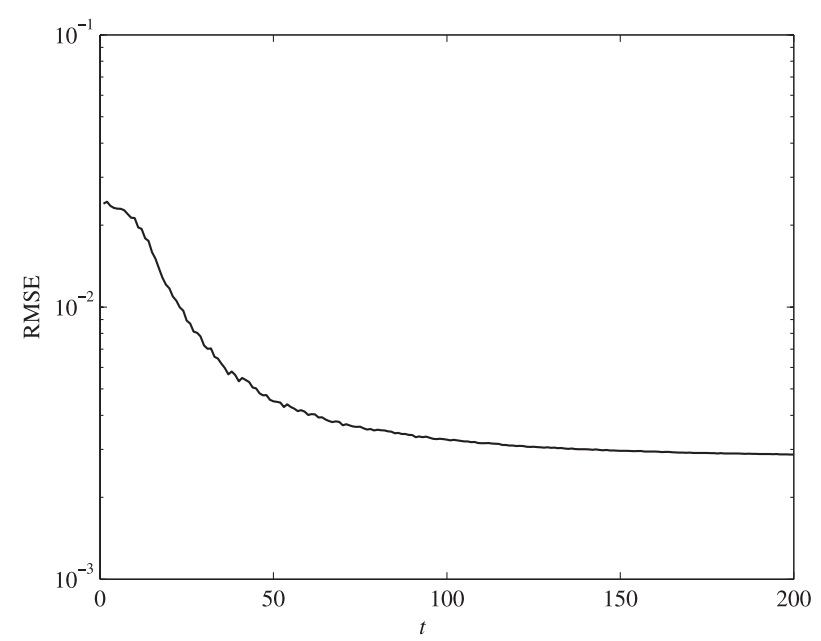

Fig. 2 RMSE of $f_{t}^{(i m a x)}$ versus $t$ of the proposed MPFD with the AVR.

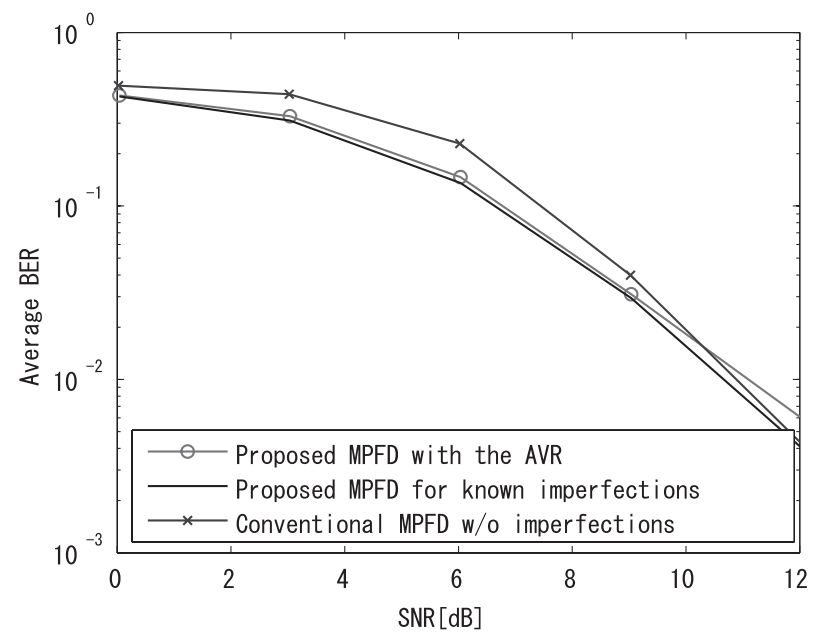

Fig.3 BER performance versus SNR of the conventional and proposed MPFDs with the computational complexity reduction.

each detector only conducts their resampling step when $M_{t}^{\text {eff }}<0.3 M$. From the figure, we can see that the MPFD with the AVR dose not lose its performance like the other MPFDs even if the resampling step is partially omitted. Hereafter, the reduction technique is employed for all the detectors with $M_{t}^{\text {eff }}<0.3 M$.

Next, in order to evaluate the efficiency of the proposed method, we test the BER performances of the MPFD with the AVR and the standard PFD versus the number of the particles $M$ where the SNR is set to be $12 \mathrm{~dB}$. In Fig. 4, it is clear that the MPFD with the AVR outperforms the standard PFD for a given number of particles. Moreover, the proposed MPFD quickly improve its performance with a small number of particles, say $M=50$ or 100 . On the efficiency, it is also interesting to compare the performance of these detectors for given computational complexity. However, a fair comparison of the standard PFD and the proposed MPFD such as by equating the number of floating-point operations (flops) faces many problems and is often useless. This is due 


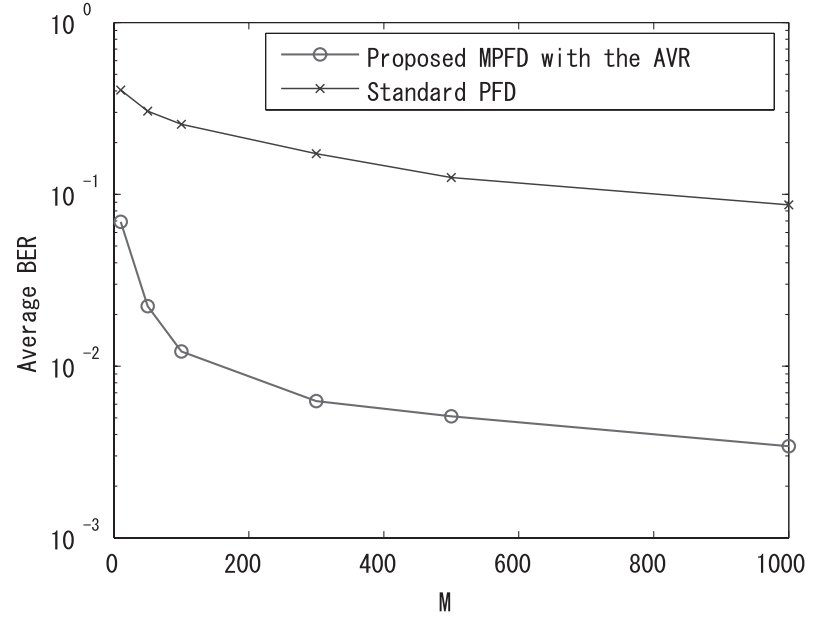

Fig. 4 BER performance versus the number of particles of the proposed MPFD and the standard PFD.
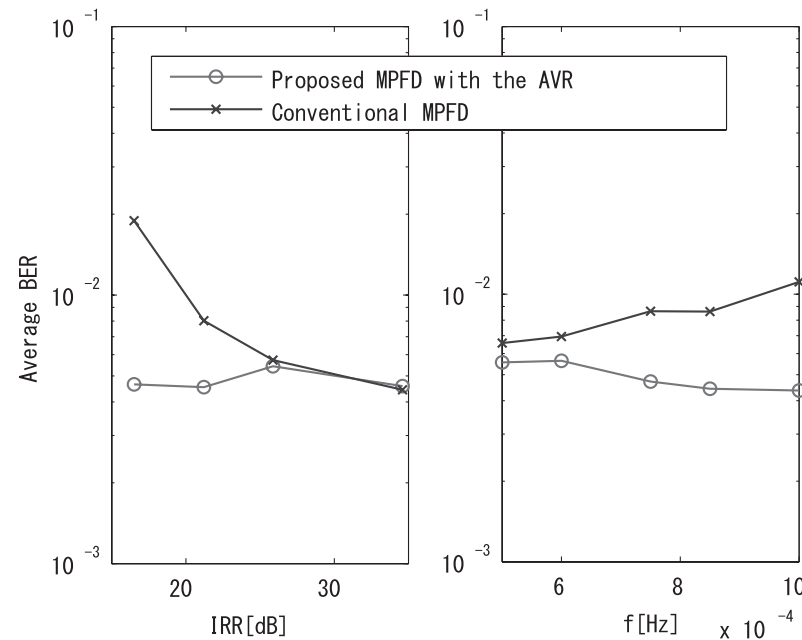

Fig. 5 BER performance versus the (a) IRR and (b) the CFO parameter $f$ of the proposed MPFD and the proposed MPFD with the AVR.

Table 1 Simulation settings for the IQI parameters

\begin{tabular}{lrrrr}
\hline $\mathrm{IRR}[\mathrm{dB}]$ & $\epsilon_{\mathrm{tx}}$ & $\epsilon_{\mathrm{rx}}$ & $\phi_{\mathrm{tx}}$ & $\phi_{\mathrm{rx}}$ \\
\hline 34.5 & 0.01 & 0.005 & 0.01 & 0.005 \\
25.8 & 0.04 & 0.01 & 0.04 & 0.01 \\
21.2 & 0.06 & 0.02 & 0.06 & 0.02 \\
16.5 & 0.08 & 0.04 & 0.08 & 0.04 \\
\hline
\end{tabular}

to the facts that some steps in the algorithm, such as generating random variables, cannot easily measured in flops and moreover the actual computational time largely depends on their implementation, such as cache boundaries and locality of the references [6]. Experimentally, the standard PFD with $M=1000$ takes 1.1 times longer computational time than the proposed MPFD with $M=300$ in our simulation and thus, from Fig. 4, it is possible to conclude that the proposed MPFD is much more efficient than the standard PFD for given computational complexity.

In addition, we further investigate the impact of each

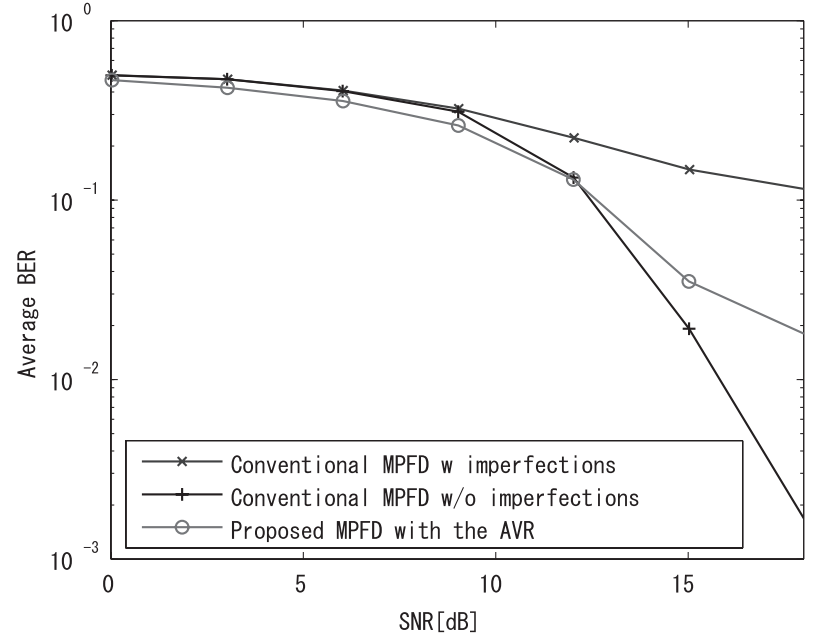

Fig. 6 BER performance versus SNR of the conventional and proposed MPFDs in the 8-DPSK modulated system.

analog imperfection on the resulting BER. Fig. 5(a) represents the BER performance versus the Tx and Rx IRR of the proposed and conventional MPFDs where the CFO is $f=0$ and $\mathrm{Tx}$ and $\mathrm{Rx}$ IQI parameters are set to be as in Table 1. On the other hand, Fig. 5(b) shows the BER performance versus the CFO parameter $f$ while the IQI parameters $\epsilon_{\mathrm{tx}}=\epsilon_{\mathrm{rx}}=\phi_{\mathrm{tx}}=\phi_{\mathrm{rx}}=0$. From the figures, in both cases of the IQIs and the CFO, we can see the significant performance deterioration of the conventional MPFD with increasing imperfections and the effectiveness of the proposed MPFD.

Finally, we test the proposed method with higher modulation level. Fig. 6 shows the BER performance of the proposed MPFD with the AVR and the conventional MPFD with or without analog imperfections where differential coded 8PSK (8-DPSK) modulation scheme is employed. In this time, we choose the discount factor $v=0.7$. Priors for the analog imperfection parameters are set to be (54), (53) and (55). Such a degree of the imperfections is relatively severe for the 8-DPSK modulated system, however the proposed MPFD still achieves much better performance compared with the conventional MPFD as in Fig. 6.

\section{Conclusions and Future Works}

In this paper, by employing the WL modeling and the AVR technique, we have applied the computational efficient MPF to the blind detection problem in the presence of the Tx and Rx IQIs and the CFO. We have shown the effectiveness of the proposed MPFD via computer simulations. The proposed MPFD can efficiently avoid the serious performance degradation due to imperfection of analog front-ends which is unavoidably occurs in realistic wireless devices.

In practice, an application of the MPFD is still limited due to its computational burden. Meanwhile, as we have shown, the MPFD is fairly easy to improve the robustness to non-linear signal distortions such as analog imperfections. 
For future works, we plan to compare the MPFD and other blind approaches based on, such as, subspace method or higher order statistics in terms of computational complexity with the additional costs for coping with non-linear distortions and this will give some contributions for practical use of $\mathrm{PF}$ in wireless communications.

\section{References}

[1] N. Gordon, D. Salmond, and A. Smith, "Novel approach to nonlinear/non-Gaussian Bayesian state estimation," IEE Proc. F., Radar and Signal Process., vol.140, no.2, pp.107-113, 1993.

[2] A. Doucet, J. Freitas, and N. Gordon, eds., Sequential Monte Carlo Method in Practice, Springer-Verlag, New York, 2000

[3] M.S. Arulampalam, S. Maskell, N. Gordon, T. Clapp, D. Sci, T. Organ, and S.A. Adelaide, "A tutorial on particle filters for online nonlinear/non-Gaussian Bayesian tracking," IEEE Trans. Signal Process., vol.50, no.2, pp.174-188, Oct. 2002.

[4] G. Casella and C. Robert, "Rao-Blackwellisation of sampling schemes," Biometrika, vol.83, no.1, pp.81-94, Dec. 1996.

[5] A. Doucet, N. Gordon, and V. Krishnamurthy, "Particle filters for state estimation of jump Markov linear systems," IEEE Trans. Signal Process., vol.49, no.3, pp.613-624, March 2001.

[6] R. Karlsson, T. Schon, and F. Gustafsson, "Complexity analysis of the marginalized particle filter,” IEEE Trans. Signal Process., vol.53, no.11, pp.4408-4411, Nov. 2005.

[7] J. Miguez and P. Djuric, "Blind equalization by sequential importance sampling," Proc. IEEE International Symposium on Circuits and Systems (ISCAS), pp.845-848, Scottsdale, May 2002.

[8] P. Djuric, J. Kotecha, J. Zhang, Y. Huang, T. Ghirmai, M. Bugallo, and J. Miguez, "Particle filtering," IEEE Signal Process. Mag., vol.20, no.5, pp.19-38, Sept. 2003.

[9] J. Miguez and P. Djuric, "Blind equalization of frequency-selective channels by sequential importance sampling," IEEE Trans. Signal Process., vol.52, no.10, pp.2738-2748, 2004.

[10] G. Fettweis, M. Lohning, D. Petrovic, M. Windisch, P. Zillmann, and W. Rave, "Dirty RF-A new paradigm," Int'l J. Wireless Information Networks, vol.14, no.2, pp.133-148, June 2007.

[11] B. Razavi, RF Microelectronics, Prentice Hall, 1982.

[12] B. Picinbono and P. Chevalier, "Widely linear estimation with complex data,” IEEE Trans. Signal Process., vol.43, no.8, pp.2030-2033, Aug. 1995.

[13] B. Picinbono, "Second-order complex random vectors and normal distribution," IEEE Trans. Signal Process., vol.44, no.10, pp.26372640, Oct. 1996.

[14] M. Pitt and N. Shephard, "Filtering via simulation: Auxiliary particle filter," J. Amer. Statist. Assoc., vol.94, pp.590-599, June 1999.

[15] J. Carpenter, P. Clifford, and P. Fernhead, "An improved particle filter for non-linear problems," IEE Proc. Radar and Sonar Navigation, vol.146, pp.2-7, 1999.

[16] A. Doucet, S.J. Godsill, and C. Andrieu, "On sequential MonteCarlo sampling methods for Bayesian filtering," J. Statist. Comput., vol.10, no.3, pp.197-208, 2000.

[17] C. Bishop, Pattern Recognition and Machine Learning, SpringerVerlag, New York, 2006.

[18] R. Chen and J. Liu, "Mixture Kalman filters," J.R. Statist. Soc. B vol.62, pp.493-508, 2000.

[19] M. Valkama, M. Renfors, and V. Koivunen, "Advanced methods for I/Q imbalance compensation in communication receivers," IEEE Trans. Signal Process., vol.49, no.10, pp.2335-2344, Oct. 2001.

[20] J. Tubbax, A. Fort, L. van der Perre, S. Donnay, M. Engels, M. Moonen, and H. de Man, "Joint compensation of IQ imbalance and frequency offset in OFDM systems," Proc. IEEE Global Telecommunications Conference in (GLOBECOM), pp.2365-2369, San Francisco, Dec. 2003.

[21] P. Schreier and L. Scharf, "Second-order analysis of improper com- plex random vectors and processes," IEEE Trans. Signal Process., vol.51, no.3, pp.714-725, March 2003.

[22] Y. Huang and P. Djuric, "A hybrid importance function for particle filtering," IEEE Signal Process. Lett., vol.11, no.3, pp.404-406, March 2003.

[23] Y. Huang and P. Djuric, "A blind particle filtering detector of signals transmitted over flat fading channels," IEEE Trans. Signal Process. vol.52, no.7, pp.1891-1900, 2004.

[24] R. Douc, O. Cappe, and E. Moulines, "Comparison of resampling schemes for particle filtering," Proc. Image and Signal Processing and Analysis (ISPA), pp.64-69, Nanjing, Sept. 2005.

[25] A. Kong, J. Liu, and W. Wong, "Sequential imputations and Bayesian missing data problems," J. Amer. Statist. Assoc., vol.89, no.425, pp.278-288, 1994

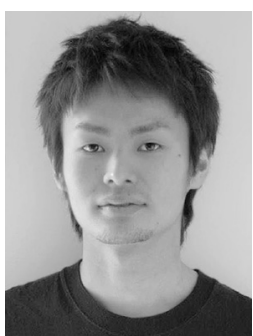

Yuki Yoshida received the B.E. and M.Info. degrees in Systems Science from Kyoto University, Kyoto, Japan, in 2004 and 2005, respectively. He is currently working towards a Ph.D in the Department of Systems Science, Graduate School of Informatics, Kyoto University.

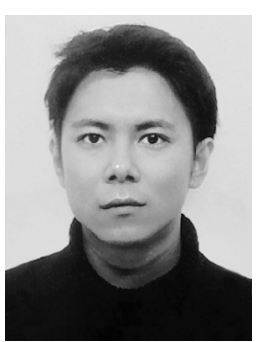

Kazunori Hayashi received the B.E., M.E. and $\mathrm{Ph} . \mathrm{D}$. degrees in communication engineering from Osaka University, Osaka, Japan, in 1997, 1999 and 2002, respectively. Since 2002, he has been with the Department of System Science Graduate School of Informatics, Kyoto University. He is currently an Assistant Professor there. His research interest include digital signal processing for communications systems.

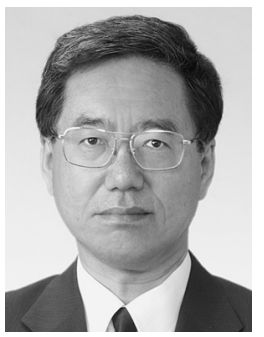

Hideaki Sakai received the B.E. and D.E degrees in applied mathematics and physics from Kyoto University, Kyoto, Japan, in 1972 and 1981, respectively. From 1975 to 1978, he was with Tokushima University. He is currently a Professor in the Department of Systems Science, Graduate School of Informatics, Kyoto University. He spent 6 months from 1987 to 1988 at Stanford University as a Visiting Scholar. His research interests are in the areas of adaptive and statistical signal processing. He served as an associated editor of IEEE Trans. Signal Processing from Jan. 1999 to Jan. 2001 and an IEEE Fellow from 2007.

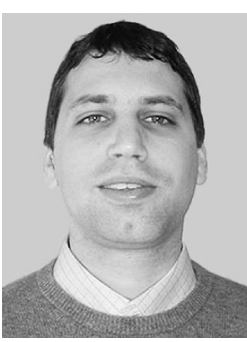

Wladimir Bocquet received the B.E. degree in communication engineering from ENST de Bretagne in 2000, the M.E. degree from University of Rennes in 2000, and Ph.D. in informatics from Kyoto University, Japan in 2008. He joined Fujitsu Laboratories Ltd. in 2000. Since 2005, he has been with France Telecom R\&D 